\title{
Susanne Reichlin
}

\section{Ordnungstransformation. Der Weltlohn}

Ordnung kann zwar als „geregelter (d.h. nicht-beliebiger) Zusammenhang von diesem und jenem “' definiert werden, doch klärt eine solche Definition einen entscheidenden Punkt nicht, nämlich kraft welcher Normen, Gesetzlichkeiten oder Konventionen ein solcher geregelter Zusammenhang besteht. Sind es rechtliche Vorgaben, Naturgesetze oder bloße Gewohnheiten, die den geregelten Zusammenhang erzeugen? Sind die Regeln implizit oder explizit definiert und wie und in welcher Form werden Irregularitäten sanktioniert? Gerade in den Geschichtsund Literaturwissenschaften wird der Ordnungsbegriff häufig für ,geregelte $\mathrm{Zu}$ sammenhänge' benutzt, deren Regeln nicht kodifiziert oder explizit festgelegt sind. Doch was begründet in einem solchen Fall die Regelmäßigkeit? ${ }^{2}$ Die Kultursoziologie kann hier, wie die Frühneuzeithistorikerin Barbara STOLLBERG-RILINGER gezeigt hat, wichtige Anregungen geben. Viele soziale Ordnungen gründen auf Erwartungs-Erwartungen: ${ }^{3}$ Der Einzelne erwartet, dass auch die anderen Beteiligten eine bestimmte soziale Ordnung, beispielsweise eine Rangordnung, voraussetzen. Wenn er mit seinen Erwartungs-Erwartungen richtig liegt und sich diesen entsprechend verhält, bestätigt er wiederum die Erwartungen der anderen und stabilisiert die entsprechende Ordnung. Der Inszenierung von Ordnung kommt

1 WALDENDFELS, Bernhard: Ordnung im Zwielicht, Frankfurt a. M. 1987, S. 17.

2 Prominent z. B. GrubmÜLLER, Klaus: Die Ordnung, der Witz und das Chaos. Eine Geschichte der europäischen Novellistik im Mittelalter: Fabliau - Märe - Novelle, Tübingen 2006, S. 113126. Vgl. dazu meine Kritik in REICHLIN, Susanne: Ökonomien des Begehrens, Ökonomien des Erzählens. Zur poetologischen Dimension des Tauschens in Mären, Göttingen 2009 (Historische Semantik 12), S. 16-19.

3 Stollberg-Rilinger, Barbara: Des Kaisers alte Kleider. Verfassungsgeschichte und Symbolsprache des Alten Reiches, München 2008, S. 9 f. Sie spricht von „institutionellen Ordnungen“ (S. 9); häufig ist auch von „symbolischen Ordnungen“ oder „Institutionen“ die Rede; vgl. zum kultursoziologischen Hintergrund von STOLLBERG-RILINGERS Thesen: REHBERG, Karl-Siegbert: Institutionenwandel und Funktionsveränderung des Symbolischen. In: Institutionenwandel. Hrsg. von Gerhard GöHLER, Opladen 1997 (Leviathan Sonderheft 16), S. 94-118; DERS.: Die stabilisierende ,Fiktionalität‘ von Präsenz und Dauer. Institutionelle Analyse und historische Forschung. In: Institutionen und Ereignis. Über historische Praktiken und Vorstellungen gesellschaftlichen Ordnens. Hrsg. von Reinhard BLÄNKNER/Bernhard JUSSEN, Göttingen 1998 (Veröffentlichungen des Max Planck Instituts für Geschichte 138), S. 381-407.

Prof. Dr. Susanne Reichlin, Ludwig-Maximilians-Universität München, Institut für Deutsche Philologie, Schellingstr. 3, 80799 München, susanne.reichlin@germanistik.uni-muenchen.de 
dabei eine wichtige Funktion zu. Soziale Ordnungen müssen durch Rituale, Tischordnungen usf. sichtbar gemacht werden, damit sie Erwartungen und Erwartungs-Erwartungen normativ steuern. Sie erzeugen so eine „Aura der Notwendigkeit“. ${ }^{4}$ Dies bedeutet jedoch keineswegs, dass solche Ordnungen nicht wandelbar wären. Vielmehr lassen sich Ordnungen gerade deshalb verändern, weil gleichbleibende Ordnungsinszenierungen (wie Tischordnung oder Rituale) den Eindruck von Dauer und Stabilität schaffen.

Das hier beschriebene Verständnis von Ordnung setzt die Geregeltheit einer Ordnung nicht voraus, sondern fragt, wie Regelmäßigkeiten und damit Ordnungen erzeugt und auf Dauer gestellt werden. Dabei wird angenommen, dass Ordnungen performativ bestätigt werden müssen. Nur wenn die Erwartungen bekräftigt werden, bleibt die Ordnung erhalten. Zugleich handelt es sich um einen prozessualen Ordnungsbegriff. Ordnungen konstituieren und wandeln sich abhängig von kollektiven Erwartungen und Praktiken. Sie sind damit per se zeitlich. Doch wann handelt es sich um eine, wann um mehrere Ordnungen? Häufig hängt dies vom gewählten Blickwinkel, von Rahmungen und Fokussierungen ab: Je größer der Ausschnitt und je weniger gerahmt soziale Phänomene betrachtet werden, umso mehr werden konkurrierende oder sich überlagernde Ordnungen bzw. Ordnungsinszenierungen sichtbar und umso weniger scheinen die Phänomene in einer Ordnung aufzugehen. Erscheinen dagegen eine Vielzahl von Phänomenen als Teil einer alles umfassenden Ordnung, so gründet dies auf Fokussierungs- und Harmonisierungsbestrebungen. ${ }^{5}$

Auch in literarischen Texten ist häufig unklar, ob wir es mit einer oder mehreren Ordnungen zu tun haben. Ebenso werden erzählte Ordnungen höchst selten benannt oder begründet. Stattdessen indizieren Erzählungen soziale Ordnungen durch das exemplarische Vorführen von Unterordnung, durch das Sanktionieren von Ordnungsvergehen oder durch Parallelen zwischen sozialen und natürlichen Ordnungen. Auch mittels Ordnungsindikatoren wie Kleidern oder mittels der Figurenerwartungen können Erzählungen funktionierende soziale Ordnungen darstellen. Sie erzählen aber auch gerne vom Scheitern von Ordnungserwartungen oder wie aus den Trümmern alter Ordnungen neue entstehen. Erzählte Ordnungen sind deshalb als Repräsentationen, Inszenierungen oder Reflexionen von sozialen Ordnungen zu verstehen. Sie bilden diese jedoch

4 Stollberg-Rilinger, Des Kaisers, S. 10 (Anm. 3).

5 Wie viele Theoretiker der Moderne nimmt auch WALDENFELS, Ordnung, S. 89-98 (Anm. 1), an, dass man in der Vormoderne davon ausgegangen ist, dass alles in einer umfassenden Ordnung aufgeht. Allenfalls ist aber auch diese These bloß das Ergebnis moderner Fokussierungsund Harmonisierungsbestrebungen und wird den historischen Gegebenheiten nicht gerecht. 
nicht nur ab, sondern sie prägen die Erwartungen der Rezipienten und sie reflektieren darüber, wie sich Ordnungen konstituieren und wandeln.

Als Ordnungen des Erzählens sind dagegen Regelmäßigkeiten auf der discours-Ebene $\mathrm{zu}$ verstehen (Erzählstrukturen, gattungstypische Erzählmuster usf.). Sie gehen den erzählten sozialen Ordnungen voran und prägen, wie diese wahrgenommen werden. Sie können die erzählte soziale Ordnung bestätigen, aber diese auch zur Disposition stellen. Es hängt somit entscheidend von den Ordnungen des Erzählens ab, ob und wie Erzählungen die Erwartungen der Rezipienten prägen.

Neben diesem Ineinander von erzählter und erzählender Ordnung sind jedoch literarhistorisch auch die Ordnungssemantiken $\mathrm{zu}$ beachten, also die Begriffe, die die Quellen benutzen, um einen ,geregelten Zusammenhang $z u$ bezeichnen. Für mittelalterliche Texte ist neben den historisch-semantisch viel erforschten, aber auch umstrittenen Begrifflichkeiten von ordo ${ }^{6}$ (lat.) bzw. orden (mhd.) der welt-Begriff von großem Interesse. Mundus oder welt bezeichnen allerdings meist keine positiv definierte Ordnung, sondern eine, die sich durch ihre corruptio, d. h. den Abfall von der göttlichen Ordnung auszeichnet. ${ }^{7}$ Dieser Weltbegriff steht in der langen Tradition des Contemptus Mundi-Diskurses, ${ }^{8}$ in dem die Welt als ein defizientes Diesseits entworfen wird. Anders als der moderne Weltbegriff, der eine autarke Einheit impliziert, bleibt ein solcher Weltbegriff auf ein positives Gegenstück bezogen. ${ }^{9}$ Doch diese großen begriffsgeschichtlichen Thesen sollen hier nicht automatisch übernommen werden, sondern geben bloß die Fragerichtung vor: Welche Ordnungsreflexion lässt sich anhand des mhd. welt-Begriffs beobachten und welche Rolle spielen dabei

6 Vgl. zu den entsprechenden Schwierigkeiten JuSSEN, Bernhard: Ordo zwischen Ideengeschichte und Lexikometrie. Vorarbeiten an einem Hilfsmittel mediävistischer Begriffsgeschichte. In: Ordnungskonfigurationen im hohen Mittelalter. Hrsg. von Bernd SCHNEIDMÜLLER/Stefan WEINFURTNER, Ostfildern 2006 (Vorträge und Forschungen / Konstanzer Arbeitskreis für mittelalterliche Geschichte 64), S. 227-256. Vgl. dazu auch Funrmann, Daniela/Selmayr, Pia: Ordnen, Wissen, Verstehen. Methodische Vorüberlegungen. In diesem Band, S. 9-11.

7 BRAUn, Herrmann: Art. ,Welt'. In: Geschichtliche Grundbegriffe 7. Hrsg. von Otto BRUNNER u. a., Stuttgart 1992, S. 433-510.

8 Wirkmächtig war insbesondere Lotario dei Segni (Innozenz III): De miseria condicionis humane. Hrsg. von Robert E. LEwIS, Athens Ga. 1978 (The Chaucer library), zwischen 1190-1194. Vgl. zur longue durée des Contemptus Mundi-Diskurses u. a.: SchILLING, Michael: Imagines mundi. Metaphorische Darstellungen der Welt in der Emblematik, Frankfurt a. M. 1979 (Mikrokosmos 4), S. 86-128; KIENING, Christian: Contemptus mundi in Vers und Bild am Ende des Mittelalters. In: ZfdA 123,4 (1994), S. 409-457.

9 Braun, Welt, S. 444-450 (Anm. 7); Kern, Manfred: Weltflucht. Poesie und Poetik der Vergänglichkeit in der weltlichen Dichtung des 12. bis 15. Jahrhunderts, Berlin 2009 (Quellen und Forschungen zur Literatur- und Kulturgeschichte $54=288$ ), S. 19-203. 
die Ordnungen des Erzählens, also die Art und Weise, wie von welt erzählt bzw. über sie gesprochen wird?

Ich möchte diese Fragen an einen in fünf Handschriften überlieferten Text stellen, in dem ganz unterschiedliche Darstellungsformen zusammentreffen und der in der Forschung meist als Der Weltlohn bezeichnet wird. In einem ersten Teil beklagt ein Ich seine Sünden und adressiert die welt, die ihm falsche Werte vermittelt habe (V. 9). Im zweiten Teil transformiert sich die Ich-Rede in eine Erzählung: Das Ich begegnet einer schönen, reich gekleideten Frau. Diese entpuppt sich dank des Eingreifens eines Pilgers (Christus) als Frau Welt. Sie geht mit ihren Dienern in Flammen auf. Die Erzählung geht in eine lange Mahnrede über, in der eine Predigerstimme an das Endgericht erinnert und das geschilderte Geschehen auf die Situation des einzelnen Gläubigen bezieht.

Unser Text wurde 1934 von August $\operatorname{CLOSS}^{10}$ ediert, wobei ihm nur vier der heute fünf bekannten Handschriften vorlagen. ${ }^{11}$ Um die Ähnlichkeit zu Konrads

10 Weltlohn, Teufelsbeichte, Waldbruder. Beitrag zur Bearbeitung lateinischer Exempla in mhd. Gewande, nebst einem Anhang. Hrsg. von August Closs, Heidelberg 1934 (Germanische Bibliothek Abt 2 37). Ich zitiere im Folgenden diese Edition, die allerdings unseren Text auf nicht gerechtfertigte Weise in zwei Texte aufteilt (s. u.). Ich benutze deshalb die Sigle JC (für Des Jamers clage) und WL (für Der Weltlohn). Die Closs nicht vorliegende Hs. K wird hinzugezogen und auch sonst konnte die Edition mit den Digitalisaten abgeglichen werden; vgl. unten Anm. 11.

11 Die Karlsruher Handschrift, Badische Landesbibliothek Lichtenthal 77 (K), 1425-1435, f. 225r238v, war Closs, Weltlohn (Anm. 10), nicht bekannt. Vgl. https://digital.blb-karlsruhe.de/blbhs/ content/pageview/1248238 (Zugriff: 10.11.2020). Diese Handschrift wurde von OTто, Arnold: Der slecht weg zuo dem himelrich. Ein oberrheinisches Erbauungsbuch. Edition und Kommentar, Berlin 2005 (Texte des späten Mittelalters und der frühen Neuzeit 42) transkribiert. In Hs. K fehlt ein substantieller Teil der Mahnrede (WL 337-388). Die Auslassung bewirkt eine Störung im Text; deshalb ist eher ein Versehen als eine gezielte Textänderung zu vermuten. Auch die anderen Handschriften des Textes sind im fünfzehnten Jahrhundert im Elsass oder im Rheinfränkischen entstanden: Berlin (B), Staatsbibliothek zu Berlin, Preussischer Kulturbesitz, Mgf 742, zwischen 1445-1460, f. 126r-136v; vgl. https://digital.staatsbibliothek berlin. de/werkansicht?PPN = PPN730239993\&PHYSID = PHYS_0264\&DMDID = DMDLOG_0016 (Zugriff: 10.11.2020). In B fehlt fast die gesamte Mahnrede (WL 327-668); Dresden (D), Sächsische Landesbibliothek, Ms. Dresd. M 60, zwischen 1427-1435, f. 70v-82r, Werkstatt Diebold Lauber, ist „vermutlich die älteste Handschrift aus dem Lauberkreis“: vgl. Katalog der deutschsprachigen illustrierten Handschriften des Mittelalters. Begonnen von Hella FRÜHMORGEN-Voss und Norbert H. OTT. Bd. 6. Hrsg. von Ulrike BodEMANN u. a., München 2015, S. 14; vgl. https://digital.slub-dres den.de/werkansicht/dlf/8957/148/0/ (Zugriff: 10.11.2020); Düsseldorf (Dü), Universitäts- und Landesbibliothek, Hs. F 55, zwischen 1460-1480, f. 51v-64v; vgl. http://digital.ub.uni-duessel dorf.de/ms/content/pageview/7295925 (Zugriff: 10.11.2020); Paris (P), Bibliothèque nationale de France, Ms. allem 117, 15. Jh., f. 87v-102v; vgl. https://gallica.bnf.fr/ark:/12148/btv1b10037664j/ f106.image (Zugriff: 10.11.2020); zu den Handschriften vgl. BoDEMANN u.a., Katalog, S. 13-18 
von Würzburg Der Welt Lohn zu unterstreichen, gab er der Erzählung den Titel Weltlohn. ${ }^{12}$ Doch unterscheidet sich nicht nur das Ende des narrativen Teils signifikant von der Erzählung Konrads sowie den damit zusammenhängenden lateinischen Exempla ${ }^{13}$, sondern auch die Rahmung ist eine andere: Während wir bei Konrad einen klar konturierten Protagonisten haben, von dem in der dritten Person erzählt und der zur Umkehr bewegt wird, beginnt unser Text mit der Reue eines Ich über sein früheres sündiges Leben (JC 1f.). Die Umkehr hat also bereits vor dem Erzählbeginn stattgefunden. Die Begegnung mit der Frau Welt geschieht als Antwort auf die Frage Waz wil dir got ze důne geben? (JC 50). Allerdings ist unklar, ob in der daran anschließenden Ich-Erzählung von derselben Ich-Instanz ausgegangen werden kann. ${ }^{14}$ Denn im zweiten Teil fehlen Bezugnahmen zum Ich des ersten Teils ${ }^{15}$ und das Ich ist vor allem Beobachter und Zeuge des Geschehens. ${ }^{16}$ Ein Prediger-Ich im dritten Teil, der Mahnrede,

(Anm. 11), sowie EICHENBERGER, Nicole: Vom Sünder und der verlorenen Frau. Erscheinungsformen einer erbaulichen Kurzerzählung - Konstruktion und Rezeptionsentwürfe. In: Lesevorgänge. Prozesse des Erkennens in mittelalterlichen Texten, Bildern und Handschriften. Hrsg. von Eckart C. LuTZ u. a., Zürich 2010 (Medienwandel - Medienwechsel - Medienwissen 11), S. 359-385, hier S. 383f.; Отто, der slecht weg, S. 16-35 (Anm. 11).

12 Eichenberger, Sünder, S. 365 (Anm. 11), gab der Erzählung dagegen 2010 den Titel Vom Sünder und der verlorenen Frau, um mit dem neuen Titel die Distanz zu Konrad von Würzburg zu betonen. Mir scheint die Diskussion um den Titel wenig ergiebig, ich bleibe bei Der Weltlohn, da sich dieser Titel in der Forschung eingebürgert hat.

13 PRIEBSCH, Robert: ,Abschied von der Welt' (Lachmann, 100, 24; Paul, 91). In: The modern language review (1918), S. 465-473, hier S. 469f., gibt einen Überblick über die lateinische Überlieferung des Exempels (eine Fassung ist im Rahmen der Gesta Romanorum überliefert) und er druckt auch zwei Fassungen ab. Im Unterschied zu unserer Erzählung zeigt die Frau in diesen beiden Fassungen freiwillig ihren Rücken und nennt ihren Namen (gloria mundi bzw. seculum). In der Fassung der Gesta Romanorum wird auch die Umkehr des Protagonisten geschildert.

14 Anders dagegen EICHENBERGER, Sünder, S. 365 f. (Anm. 11), die die „überall präsente Erzählerfigur“ als das „verbindende Element“ des Textes betrachtet.

15 Das Ich ist anfänglicher Dialogpartner der Frau und nimmt - anders als bei Konrad - das Dienstangebot der Frau weder freudig an, noch lehnt es entschlossen ab (WL 57-61). Danach verschwindet es geradezu aus dem Geschehen. Fokalisierungen oder Innensicht fehlen gänzlich. Erst am Ende des narrativen Teils gibt es kommentierende Verse (WL 279-287), die allerdings mit einem predigenden Sprechgestus gesprochen sind, der auf die Mahnrede vorausweist und sich nur schlecht mit der unschlüssigen Stimme, die mit Frau Welt gesprochen hat, vereinbaren lässt.

16 Erzähltes und erzählendes Ich sind voneinander losgelöst. Dies ist typisch für viele mittelalterliche Ich-Erzählungen; vgl. dazu den guten Überblick bei GLAUCH, Sonja/PHILIPOWSKI, Katharina: Vorarbeiten zur Literaturgeschichte und Systematik vormodernen Ich-Erzählens. In: Von sich selbst erzählen: Historische Dimensionen des Ich-Erzählens. Hrsg. von DENS., Heidelberg 2017 (Studien zur historischen Poetik 26), S. 1-61, hier S. 4; SPEARING, A. C.: Medieval Autographies. The „I“ of the Text, Notre Dame, Ind. 2012 (The Conway lectures in medieval 
unterscheidet sich wiederum von den vorangehenden Ich-Instanzen dadurch, dass es ausgehend von der (ihm bekannten, aber nicht selbst erfahrenen) Erzählung mahnt und belehrt. ${ }^{17}$ Wir haben somit kein Bekehrungsnarrativ, sondern eine Erzählung in der ersten Person in der Mitte, die durch eine Rede eines Ich am Beginn und eine Rede am Ende gerahmt und mit Deutung versehen wird.

Handelt es sich also gar nicht um einen Text mit drei Teilen, sondern um drei lose verknüpfte eigenständige Texte? Die Handschriften gestalten den $\mathrm{Zu}$ sammenhang der drei Teile unterschiedlich. Wie Nicole EICHENBERGER gezeigt hat, präsentiert die Karlsruher Handschrift den gesamten Text (Ich-Rede, narrativer Mittelteil und Mahnrede) fast ohne Binnengliederung. ${ }^{18}$ Die anderen vier Handschriften unterbrechen den Text dagegen mit Rubriken und z. T. Bildern (D, Dü, B): Sie gliedern ihn jedoch nicht in die kompositorisch naheliegenden drei Teile, sondern heben den Beginn des narrativen Mittelteils bzw. das Auftreten der Frau Welt, ihre Enthüllung sowie innerhalb der Mahnrede die vier pfiffer (apokalyptische Bläser bzw. Engel) hervor. ${ }^{19}$ Die textinternen Rubriken sind

studies). Unser Text gehört zu der Familie von spätmittelalterlichen Ich-Erzählungen, die dominant wissensvermittelnd sind. Der Ich-Erzähler präsentiert eine partikulare Erfahrung, die zugleich eine exemplarische ist; vgl. GLAUCH/PHILIPOWSKI, Vorarbeiten, S. 41-45 (Anm. 16). Allenfalls unterscheidet ihn die Mehrstimmigkeit der Ich-Aussagen (siehe Anm. 17) von anderen Texten dieser Textgruppe (z. B. Minnereden).

17 Auch in der Mahnrede sind mehrere Stimmen hörbar, mit Sicherheit können ein exemplarisches Sünder-Ich und eine Predigerstimme voneinander unterschieden werden (siehe unten Abs. IV.)

18 In der Karlsruher Hs. K trägt der Text die Überschrift: hie hebet sich an des iamers clage die / man horet an dem iungesten dage (f. 225r). Die anderen Handschriften weisen ähnliche Überschriften auf: Dis ist des jomers clage am jungesten tage (Hs. P, f. 87v), die den Fokus auf den ersten Teil des Textes legen, in denen ein Ich sein bisheriges sündiges Leben bereut. Der Text der Handschrift K wird einzig durch vier Paragraphenzeichen gegliedert (WL 277, 299, 480, 617 bzw. f. 231v, 234r, 235r, 237v). Die Funktion der Zeichen (geht es um Gliederung oder Hervorhebung) ist nicht ganz klar; vgl. dazu unten Anm. 35.

19 In allen Handschriften (außer K) finden sich an denselben Stellen (mit der Abweichung weniger Verse) textinterne Rubriken mit ähnlichem Wortlaut: Nach JC 52; Von der scho̊nen verlornen fröwen (Hs. D, f. 71v); nach WL 222; Hs. D, f. 75r (Abb. 1): wie die scho̊ne fröwe slangen und kretten ossent / und su ire scho̊ne verlorn het; auch in der Berliner Hs., f. 133v, findet sich die Rubrik: Dis saget wie die schöne frouwe die krötten und / slangen ossent und ir schöne verlorn hat. Das entsprechende Bild dazu (Abb. 2) findet sich jedoch erst auf der nächsten Seite (f. 134r). Auch innerhalb der Mahnrede findet sich eine Rubrik samt Bild, meist nach WL 363: Dis sint die vier engelschen pfiffer die alle / sünder von dem tode heissent uff ston (Hs. P, f. 96r). Die Pariser Handschrift weist ähnliche textinterne Rubriken wie die Bilderhandschriften auf, ist aber selbst ohne Bilder; vgl. dazu EICHENBERGER, Sünder, S. 375f. (Anm. 11). 
deshalb wohl weniger als Textgliederung und mehr als Bildüberschriften zu verstehen. ${ }^{20}$ Allerdings folgt in zwei Handschriften (D und B) im Anschluss an unseren Text eine Paraphrase der Zehn Gebote (20 Verse) und die Erzählung Teufelsbeichte, die jeweils durch eine gleich gestaltete Überschrift mit Bild eingeleitet werden. ${ }^{21}$ Die Grenzen zwischen Einzeltext und Textkompilation sind somit nicht immer eindeutig zu bestimmen. ${ }^{22}$ Doch gerade dies erlaubt uns $\mathrm{zu}$ fragen, wie in mehreren Textteilen ähnliche Ordnungen mit unterschiedlichen Darstellungsmitteln entworfen werden.

In allen drei Teilen unseres Textes geht es um die welt als Bezeichnung für einen geregelten Zusammenhang, der sich von der göttlichen Heilsordnung unterscheidet. Das, was mit welt bezeichnet wird, und das, was davon ausgehend als erzählte Ordnung der welt dargestellt wird, verändert sich innerhalb des Textes immer wieder. Dies hängt zum einen mit den Ordnungen des Erzählens zusammen: Je nachdem, ob die welt personifiziert wird oder nicht, ob erzählt wird oder in Form einer Rede über den Zustand der welt nachgedacht wird, sind die dahinterstehenden Welt- und Ordnungskonzepte andere. Zum anderen wird die Ordnung der welt insbesondere im narrativen Mittelteil aber auch als eine sich transformierende gezeigt.

Im Folgenden soll deshalb entlang der drei Teile des Textes untersucht werden, wie die Ordnung der welt sowie die mit ihr konkurrierende und ihr übergeordnete christliche Ordnung konzipiert und dargestellt werden. Dazu wird im Abschnitt I. analysiert, wie die welt in der Ich-Rede als Werteordnung entworfen wird, wobei die an die Werte geknüpften Erwartungen eine zentrale Rolle spielen.

20 So EICHENBERger, Sünder, S. 373f. (Anm. 11). Vgl. zu dieser insbesondere für die LauberWerkstatt typischen Gliederungsform auch PALmER, Nigel F.: Kapitel und Buch. Zu den Gliederungsprinzipien mittelalterlicher Bücher. In: Frühmittelalterliche Studien 23 (1989), S. 43-88, hier S. $74 \mathrm{f}$.

21 Dresden f. 82r und Berlin f. 136v. GeISs, Jürgen: Art. ,Weltlohn‘. In: VL 10, Berlin, New York ${ }^{2} 2010$, Sp. 838-840, hier Sp. 838, vermutet, dass der Dekalog den „ursprünglichen Abschluss“ des Weltlohns gebildet habe. Dem muss man nicht folgen, da in beiden Handschriften die Teufelsbeichte mit einer gleich gestalteten Rubrik samt Bild anschließt. Vielmehr zeigt sich, dass die Textgrenzen unklar resp. offen sind. Dies gilt auch für die Grenze zwischen narrativem Mittelteil und Mahnrede (siehe unten Abs. IV).

22 Auch bei der größeren Textkompilation, zu der auch der Weltlohn gehört, bei dem sog. Oberrheinischen Erbauungsbuch, sowie beim größten Textkonglomerat dieser Sammlung, der slecht weg zů dem himelrich (in Hs. K f. 77v-199r, vgl. Отто, Der slecht weg [Anm. 11]), sind die Textgrenzen nicht klar bestimmbar; so WACHINGER, Burghart/OTTO, Arnold: Art. ,Der slecht weg“ und das ,Oberrheinische Erbauungsbuch'. In: VL 11, Berlin, New York ${ }^{2} 2010$, Sp. 1437-1442, hier Sp. 1437. 
Im II. Abschnitt soll gezeigt werden, wie im narrativen Teil die Ordnung der welt nicht wie im ersten Teil vorausgesetzt, sondern anhand von Ordnungsindikatoren (Kleider, Jahres- und Tageszeiten, Hierarchien) prozessual entwickelt wird. Dabei steht die personifizierte Frau Welt für eine defiziente oder korrupte Ordnung, die mit einer anderen, durch Christus repräsentierten Ordnung, konfrontiert wird. Diese Ordnungskonkurrenz wird im III. Abschnitt untersucht. Der IV. Abschnitt widmet sich der Mahnrede. Diese thematisiert das Verhältnis von weltlicher und göttlicher Ordnung erneut, aber nicht mehr narrativ, sondern diskursiv: Was kann eine solche Ordnungskonkurrenz für den einzelnen Gläubigen bedeuten?

\section{Weltbegriffe und Ordnungskonzepte}

Im ersten Textteil artikuliert sich die Reue des Ich im Vorwurf an die welt, sie habe das Ich mit ihrer falschen lere (JC 9) verführt, das Ich habe von ihr immer nur schande[ ] und untruwe[ ] (JC 13) erfahren. Durch diese Adressierung wird die welt als Gegenüber des Ich dargestellt - ein Gegenüber, das die Versprechen, die es macht, nicht erfüllt, und dem deshalb der dienst[ ] und das Vertrauen aufgekündigt werden: Ez ist allesamt erlogen / Waz du ie hast geheizen mir, / Davon getruwe ich nime dir (JC 40-42). Die Erfahrung, die das Ich gemacht hat, wird generalisiert und den Rezipienten als Regel präsentiert: Wer an dich pflihtet oder habet, [...] Der struchet unde vellet (JC 15, 17). Ganz im Sinne der Eingangsüberlegungen wird hier die Ordnung, für die die welt steht, auf der Ebene von Erwartungen verhandelt. Wenn sich die Rezipienten an die mit der Welt verknüpfte Werteordnung halten und entsprechende Erwartungen ausbilden, haben sie mit Enttäuschungen zu rechnen. Dabei steht das Ich mit seinen Erfahrungen für diese enttäuschten Erwartungen ein. Dem Ich selbst kommt hierbei eine ambivalente Rolle zu: Aus Sicht des Ich wird die Welt als Ordnung entworfen, von der es sich lossagen will. Für die Rezipienten ist es jedoch Teil dieser Ordnung. Seine Erfahrungen bezeugen, dass die Hoffnungen, die mit weltlichen Werten verbunden sind, enttäuscht werden. Damit werden wiederum die Erwartungen der Rezipienten an weltliche Werte minimiert und versucht, veränderte Erwartungsordnungen zu erzeugen.

Diese Ausrichtung auf die Rezipienten und ihre Erwartungen begründet auch die kreisende Struktur des ersten Textteils, der mit den Erfahrungen des Ich mit der welt beginnt (JC 1-12) und endet (JC 39-46). Dazwischen wird mit einem kurzen allegorisch-narrativen Sinnbild verdeutlicht (JC 17-34), für welche Werte die welt steht. Die hoffart (superbia) sei aus dem Himmel vertrieben worden. An- 
schließend sei ihr nur die welt als Lebensraum gebliben (JC 22, 24): Hier habe sie ihren Samen ausgesät und Laster wie Bosheit, Unkeuschheit oder untruwe hätten sich so vermehrt, dass gotes haz zu befürchten sei (JC 29, 37). In diesen Passagen wird Ordnung nicht durch Erwartungs-Erwartungen, sondern axiomatisch durch bestimmte Werte konzipiert. Die dargestellte Ordnung der welt gründet auf bestimmten, negativ konnotierten Werten wie untruwe oder Bosheit. Daneben wird in dieser Passage aber auch ein räumlicher Ordnungs- und Weltbegriff aufgerufen: welt ist ein Bereich, der sich vom himel unterscheidet (JC 23f.). Wer wie die hoffart aus dem einen Bereich verstoßen wird, der muss sich im anderen Bereich (welt) aufhalten.

In diesem ersten Teil sind somit drei welt-Begriffe erkennbar: Welt erscheint als etwas, das durch bestimmte Erwartungen erzeugt wird; sie wird sodann zu einem geregelten Zusammenhang, der auf geteilten Werten beruht, und am Ende handelt es sich um einen räumlichen Bereich. Damit gehen auch drei verschiedene Ordnungskonzepte einher: ein performatives Ordnungskonzept, das auf Erwartungs-Erwartungen beruht, ein axiomatisches, das auf Werten gründet, sowie ein räumliches Ordnungskonzept. Verknüpft und harmonisiert werden diese verschiedenen Ordnungskonzepte durch den Begriff und die Adressierung der welt. Mittels der Instanz welt werden verschiedene, kaum miteinander kompatible Ordnungskonzepte als Teil einer übergreifenden Ordnung dargestellt bzw. zu einer solchen harmonisiert.

\section{Ordnungsindikatoren und Erwartungslenkung im narrativen Mittelteil}

Es gilt nun zu untersuchen, welche Formen der Ordnungsdarstellung und welche damit verknüpften Weltbegriffe im zweiten, erzählenden Teil des Textes entwickelt werden. Wie bereits erwähnt, stehen Ich-Rede und narrative Ich-Erzählung nicht unverbunden nebeneinander, sondern aus der Ich-Rede heraus ergibt sich die visionsartige Begegnung mit der Personifikation der Frau Welt. ${ }^{23}$ Das Ich bereut die vertane Zeit (vloren zit, JC 45) und fragt sich: wie soltu nů leben? / Waz wil dir got ze důne geben? (JC 49f.). Da kommt eine frowe minneclich (WL 1) mit reicher Kleidung. Sie prahlt, dass alle (auch keiser, künige) ihr undertan seien (WL 45-49). Als das Ich nicht sogleich in den Dienst einwilligt,

23 Vgl. zu dieser auch für die Minnereden typischen Begegnung eines Ich-Erzählers mit einer Personifikation: GlaUCH/PHILIPOWSKI, Vorarbeiten, S. 16-21, 55-59 (Anm. 16). 
führt die Frau es auf einen anger wunneclich (WL 63). In topisch beschriebener Mai-Natur werden Zelte aufgestellt und finden ritterspil (WL 89) statt. Nach Speis und Trank erscheint gegen Abend ein alter Pilger. Er fordert die Frau auf, Krone und Zepter abzulegen (WL 171f.) und ihren Namen zu nennen (WL 178). Die Frau anerkennt den Pilger sogleich als ihr übergeordnet: Der here ist komen mit siner gewalt, [...] Der twinget mich uf disen tac / Daz ich die warheit sagen mů3, / [...] Ich bin die Welt geheizen (WL 192-197) ${ }^{24}$. Bevor sie ihre Kleider ablegt, charakterisiert sie sich selbst als jemanden, der die Menschen zu hoffart (WL 201), unkuscheit (WL 211) und unrechtem Gewinnstreben (wůcher und unreht gůt; WL 202) animiert hat. Ihre Rede geht aus dem ich ins wir über, wenn sie über die weltlichen Güter spricht: Daz můz uns hie zerrinnen (WL 204). Erst nach dieser Rede wird beschrieben, wie sie ihre Kleider ablegt und ihr bereits verfallener, vom Ungeziefer durchdrungener Körper sichtbar wird (Abb. 1 und 2).

Sie kann noch bekannt geben, dass ihr und ihren Dienern als lon der $\mathrm{Zu}$ gang zum Himmelreich verwehrt sei (WL 234f.), dann gehen Lichtung und Wald in Flammen auf. Die Schreie der Verdammten sind zu hören. Der Pilger verweist auf seine Wundmale und droht den Rezipienten, dass sie denselben Lohn wie die Weltdiener zu erwarten haben.

Die in der Ich-Rede bloß adressierte welt tritt somit im Mittelteil als Personifikation auf. Allerdings wird erst kurz vor ihrer Zerstörung explizit gemacht, dass es sich bei der frowe um die personifizierte Frau Welt handelt. Stärker als im ersten Textteil haben wir es somit (auf der Darstellungsebene) mit einem prozessualen Welt- und Ordnungskonzept zu tun. Diese prozessuale Ordnungsdarstellung geschieht jedoch nicht nur anhand der Personifikation, sondern auch anhand von Ordnungsindikatoren. Damit sollen Indices (Kleider, Tageszeiten, Herrschaftssymbole) bezeichnet werden, die im Text aufgerufen werden, um das Geschehen innerhalb eines geregelten Zusammenhangs zu verorten.

Nachdem der Ich-Erzähler die Frau ausführlich beschrieben hat, ordnet er das Geschehen in den Tagesablauf ein: Daz was eins morgens gegen dem tage / Vor mettin zit als ich uch sage (WL 29f.). Die Deutung der Tageszeit wird ebenfalls expliziert:

Ich gedahte: waz wil hie geschehen?

Du hast ouch dicke geho̊ret sagen,

Wanne ez schiere begunde tagen

Daz do iht ungehures si.

(WL 32-35)

24 In Hs. K steht Ich bin die welt bescheiden; zitiert nach der Transkription von Отто, der slecht weg, S. 506, V. 249 (Anm. 11). 


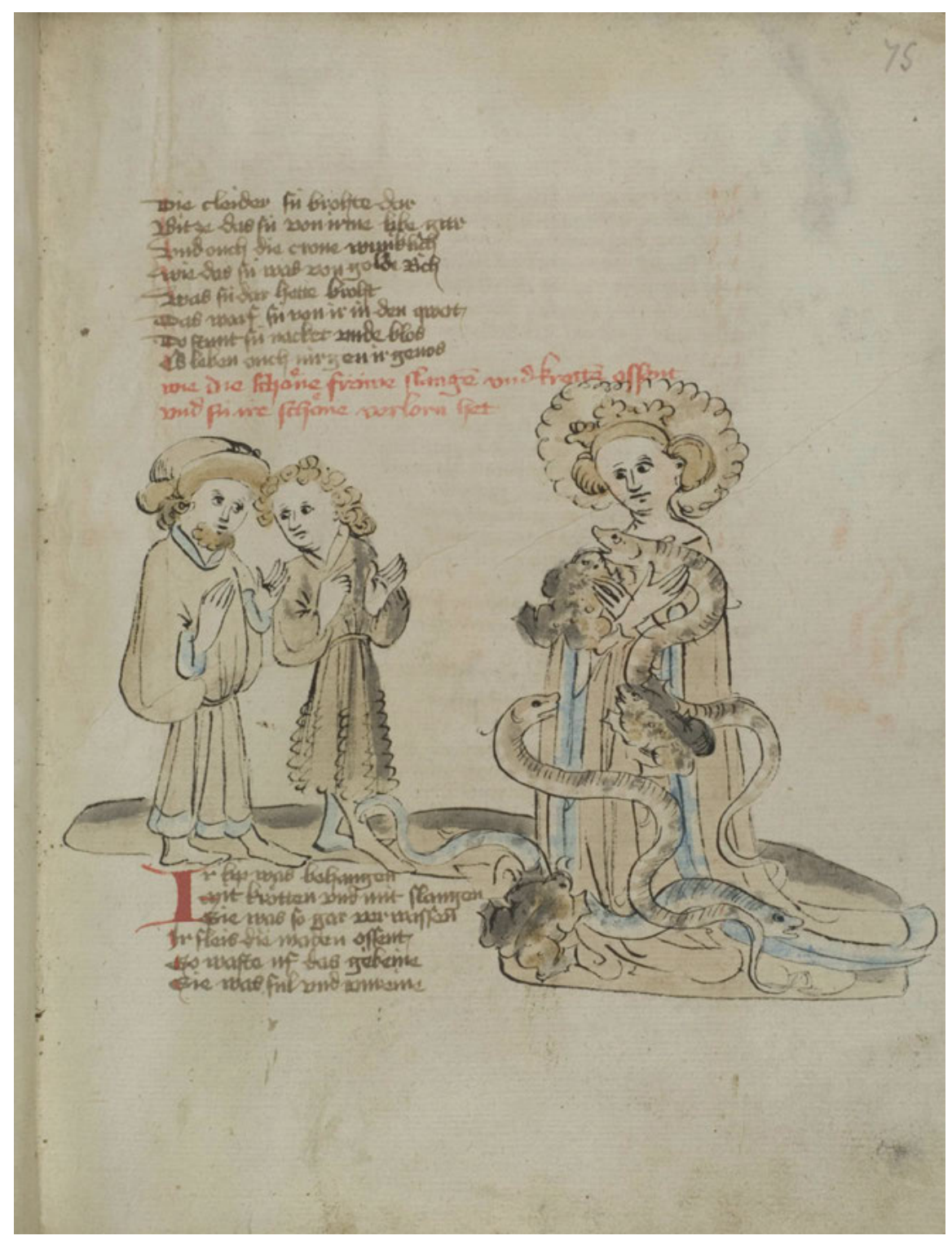

Abb. 1: Dresden, Sächsische Landesbibliothek, Ms. Dresd. M 60, f. 75r (Werkstatt Diebold Lauber). 


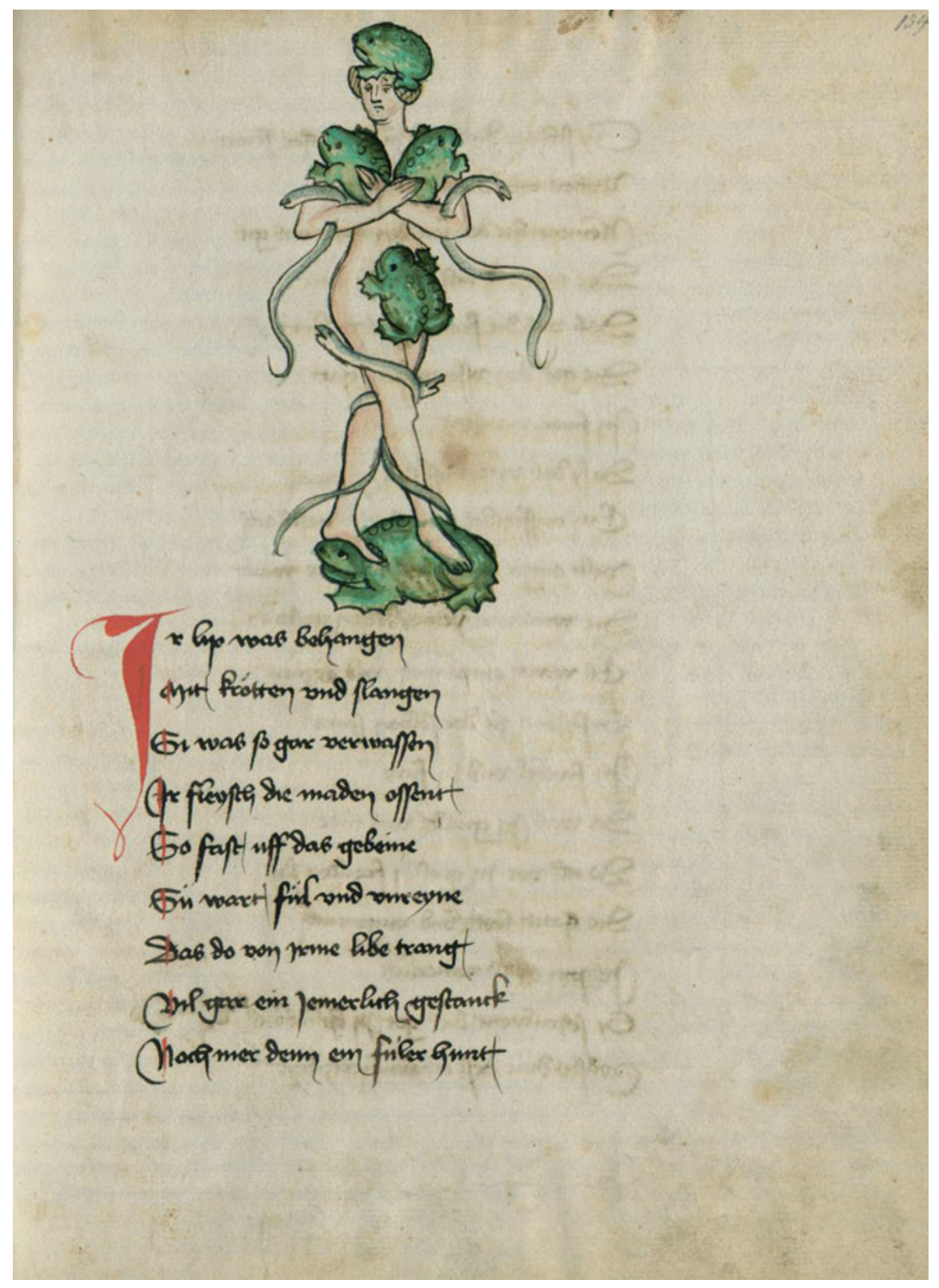

Abb. 2: Berlin, Staatsbibliothek zu Berlin, Preussischer Kulturbesitz, Mgf 742, f. 134 r. 
Das Muster des Tagesablaufs wird so mit Wertungen versehen, die helfen, das Geschehen einzuordnen: Der frühe Morgen ist eine ungehure, also eine unheimliche, zweideutige Zeit. Die Signifikanz der Tageszeit ergibt sich durch den wiederholten Verweis darauf. Später tritt der Pilger zur vesper zit auf und der Erzähler kommentiert: umb die vesper zit / So man daz abentezzen git, / Der schimpf der solte ein ende han (WL 143-145). Die Frauenfigur erscheint mit dem Tag und geht mit ihm zu Ende. Sie und die durch sie repräsentierte Ordnung werden so als vergänglich konnotiert.

Doch sind die Tageszeiten keineswegs die einzigen Ordnungsindikatoren, die im Mittelteil aufgerufen werden. Wenn die wohlgeformten Gebärden der Frau, ihr mit Edelsteinen besetzter Gürtel und ihr gefütterter Mantel beschrieben werden (WL 9-25), wird die Frau mittels dieser Indices einer höfischen Sphäre zugeordnet. Sie führt den Ich-Erzähler zu einem Turnier und Fest, also zu typischen Repräsentationsformen einer höfischen Ordnung. Die Frauen sitzen auf weißen Kissen und beobachten, Wer do beste keme dar, / Daz sie dem seiten lop und danck. / Also daz volck nach eren ranc (WL 100-102). Die Konkurrenz der Beteiligten um Ehre und Anerkennung, die sowohl mit materiellem Prunk als auch im rituellen Wettkampf (Turnier) erwirtschaftet werden können, konstituiert die dargestellte höfische Ordnung mit.

Die Frau präsentiert sich als diejenige, die dieser Ordnung vorsteht. So sagt sie: Sih umbe dich, waz man siht. / In mime dienste daz geschiht (WL 115f.) oder Mir dienet waz uf erden ist: / Keiser, künige, fürsten gar, / Grafen, frien mit ir schar (WL 44-46). ${ }^{25}$ Neben den zeitlichen und symbolischen Ordnungsindikatoren wird so auch auf ein hierarchisches Ordnungskonzept verwiesen: Die Frauenfigur besetzt die hierarchisch höchste Position und verfügt auch über die entsprechenden Machtinsignien (Zepter, Krone). Sie ist dem, was die Rezipienten als höchste Stufe der sozialen Hierarchie kennen, übergeordnet.

Diese Ordnung, der die Frau vorsteht, wird als hof [ ] (WL 166, 176), später auch als der welte hof (WL 301) bezeichnet. Die Vollkommenheit dieser Ordnung wird durch Jahreszeiten markiert: Der hof hat in der meien zit statt (WL 86) und die ausführlich beschriebene Natur ist in Feststimmung: Die Vogelstimmen bieten froudenrichen schal (WL 77) und werden als fri und wol gemüt (WL 81) bezeichnet. Die Harmonie von natürlicher und kultureller Pracht, von blühender Jahreszeit und sozialer Feststimmung bestätigt den umfassenden Geltungsanspruch der dargestellten höfischen Ordnung positiv.

25 Dies ist eine der textübergreifenden Aussagen der Frau Welt, sie findet sich auch bei Konrad von Würzburg Der Welt Lohn, V. 202-209; vgl. Konrad von Würzburg: Der Welt Lohn. Hrsg. von Reinhard BLECK. Göppingen 1991 (Litterae 112). 
Die meisten der beschriebenen Ordnungsindikatoren gehören topisch $\mathrm{zu}$ dem, was in der Forschung als ,höfische Ordnung' bezeichnet wird. Dies en détail nachzuvollziehen, hat den Vorteil, die Vielfalt der höfischen Ordnungsindikatoren sichtbar zu machen: Kleider und Herrschaftssymbole, Jahreszeiten, die Harmonie von Natur und Kultur, Hierarchien und soziale Rituale. Damit gehen selbstredend auch unterschiedliche Ordnungskonzepte einher: zeitliche, symbolische, hierarchische, räumliche usf. Die eine ,höfische Ordnung،, von der insbesondere mit Bezug auf den Artusroman häufig die Rede ist, wäre deshalb vermehrt als Ergebnis verschiedener, aber miteinander harmonisierter Ordnungsindikatoren zu verstehen. In unserer Erzählung bestätigen die Ordnungsindikatoren sich jedoch nicht nur gegenseitig, sondern die Tageszeiten unterlaufen diese Bestätigung auch. Sie betonen die Vergänglichkeit der dargestellten Ordnung von Beginn an. Betrachtet man den Mittelteil nicht isoliert, so wird die dargestellte höfische Ordnung auch durch den ersten Teil, i. e. die Rede des reuigen Ich, in Frage gestellt. Zudem wird, noch bevor der Pilger auftritt, durch einen Kommentar (WL 143-156) vorausgreifend erklärt: Der schimpf der sollte ein ende han [...] Ez ist ein krancke zůversicht, / Ir [der frouwe] hoffart weret iemer niht, / Sie můz erfulen als ein mist (WL 145, 153-155).

Die Forschung schreibt unserem Text deshalb auch im Vergleich zu anderen Frau-Welt-Erzählungen eine Tendenz zur Vereindeutigung zu: Die Erzählung verliere jegliche Spannung und Zweideutigkeit. ${ }^{26}$ Statt dies zu beklagen, ist zu betonen, dass die Aufmerksamkeit so vom ob, auf das wie verschoben wird. ${ }^{27}$ Obwohl die Rezipienten um die Defizienz der höfischen Ordnung wissen, wird diese als eine nahezu ideale entworfen. Erst danach wird der Anspruch der erzählten Ordnung intradiegetisch depotenziert. Die Erwartungen der Rezipienten an höfische Ordnungsindikatoren werden so zugleich geschürt und disqualifiziert. Es ist zu vermuten, dass eine doppelte Lesbarkeit eingeübt wird: Die Rezipienten sollen die höfischen Ordnungsindikatoren verstehen, aber die erste, höfische Deutung auf eine zweite, christliche Deutung hin überwinden.

26 EICHENBERGER, Sünder, S. 364 (Anm. 11); WitTHÖFT, Christiane: Sinnbilder der Ambiguität in der Literatur des Mittelalters. Der Paradiesstein in der Alexandertradition und die Personifikation der Frau Welt. In: Ambiguität im Mittelalter. Formen zeitgenössischer Reflexion und interdisziplinärer Rezeption. Hrsg. von Oliver Auge/Ders., Berlin 2016 (Trends in Medieval Philology 30), S. 179-202, hier S. 200.

27 Vgl. Lugowski, Clemens: Die Form der Individualität im Roman, eingel. von Heinz ScHLAFFER, Frankfurt a. M. ${ }^{2} 1994$ (stw 151), S. 40-44. 


\section{Konkurrierende und transformierte Ordnungen}

Innerhalb des narrativen Mittelteils wird also zunächst eine höfisch-irdische Ordnung entworfen, die sodann im Namen einer höheren christlichen Ordnung abgewertet wird. Ein Pilger tritt im grawe[n], wilde[n] cleit (WL 159) mit zwölf Rittern auf (WL 162-164). Er verlangt von der Frau, die Kleider samt den Herrschaftssymbolen abzulegen (WL 170-172). Sie erhält somit anders als in den meisten Erzählungen der Frau Welt-Tradition einen Gegenspieler, der sich später als Christus erweisen wird (WL 288). ${ }^{28}$ Er relativiert ihre Macht, indem er sich ihr überordnet und ihr Befehle erteilt. ${ }^{29}$ Die davor etablierte Hierarchie wird so umbesetzt. Dazu werden auch die weltlichen Herrschaftssymbole weiter genutzt: Durch das Ablegen von Zepter und Krone wird dargestellt, dass die Macht der Frau Welt begrenzt ist (WL 171f., 217).

Auch der Ordnungsindikator ,Kleidung“ wird nicht aufgegeben, sondern umcodiert: Der Pilger fordert die Frau auf, die Kleider anzuziehen, [d]ie ir von rehte sullet han (WL 182). Sie steht nackent unde bloz (WL 221) da und sichtbar wird der bereits verfallene, vom Ungeziefer zerfressene Körper. Während Frau Welt bei Konrad von Würzburg ihre verfallene Rückseite durch eine Drehung freiwillig zeigt, enthüllt sie in unserer Erzählung ihren ,wahren' Körperzustand erst auf fremdes Geheiß. Der Gegensatz von Schein und Sein, Täuschung und Wahrheit steht so im Vordergrund und nicht, wie allenfalls bei Konrad, die Am-

28 In bildlichen Darstellungen hat Frau Welt jedoch häufig ein positives Pendant: vgl. die sog. Mettener Armenbibel (BSB Clm 8201, f. 95r), in der eine figura mundi (Siebenlaster-Weib) und Benedikt einander gegenüber stehen (http://daten.digitale-sammlungen.de/0004/ bsb00040329/images/index.html?id=00040329\&groesser=\&fip=eayayztsweayaxsfsdryztsfsdrxd sydxdsydqrs\&no=7\&seite=193; Zugriff: 10.11.2020); vgl. dazu SuCKALE, Robert: Klosterreform und Buchkunst die Handschriften des Mettener Abtes Peter I. München, Bayerische Staatsbibliothek, Clm 8201 und Clm 8201d, Petersberg 2012, S. 104; CuRSCHMANN, Michael: Facies peccatorum Vir bonus. Bild-Text-Formeln zwischen Hochmittelalter und früher Neuzeit. In: Poesis et pictura. Studien zum Verhältnis von Text und Bild in Handschriften und alten Drucken. Festschrift für Dieter Wuttke zum 60. Geburtstag. Hrsg. von Joachim KNAPE/Stephan FÜSSEL, Baden-Baden 1989 (Saecula spiritalia Sonderband), S. 157-189, hier S. 176; vgl. auch die Skulpturen in der Ecknische der Annenkapelle bzw. am Südportal des Wormser Doms. Oben stehen Caritas und Fides, unten Synagoge (wohl im Sinne von Infidelitas) und Frau Welt mit einem von Kröten behangenen Rücken. Während Frau Welt einem Ritter zu ihren Füßen den Schild reicht, ist zu Füßen von Caritas ein Bettlerpaar. Vgl. dazu SEBALD, Eduard: Gotisch und romanisch? Das Hauptportal auf der Südseite des Wormser Doms. In: Der Dom zu Worms: Krone der Stadt. Festschrift zum 1000jährigen Weihejubiläum des Doms. Hrsg. von Peter KoHLGRAF u. a., Regensburg 2018, S. 61-66, hier S. 63.

29 Vgl. WL 173f.: Wa3 ir gewelte muget han, / Der müezet ir uch machen an. Die Frau akzeptiert seine Macht über sie explizit: Der here ist komen mit siner gewalt (WL 192). 
bivalenz einer von Gott geschaffenen, aber mit Sünde belasteten Welt oder die widersprüchliche Gleichzeitigkeit von lebendem und verfallendem Körper. ${ }^{30} \mathrm{Be}-$ tont wird so, dass die höfischen Kleider falsche Erwartungen wecken. Ähnlich wie bei der Hierarchie geht es darum, den Ordnungsindikator ,Kleidung“ anders zu besetzen: Der grawe, wilde Rock des Pilgers überbietet mit den damit einhergehenden Zeichen der Askese die höfische Kleidertracht positiv. Nicht die höfische Opulenz, sondern die einfache Kleidung steht für das Höherrangige. ${ }^{31}$

Gründen Ordnungen auf Erwartungen, die wiederum von Ordnungsindikatoren abhängig sind, dann ist es entscheidend, welche Geltung und Bedeutung den einzelnen Indikatoren zukommt. Solche Geltungs- und Deutungsdifferenzen werden in Der Weltlohn verhandelt. Zugleich wird auf der Handlungsebene, anhand der Personifikation der Frau Welt und ihrem Gegenspieler (Pilger), die Ordnungskonkurrenz allegorisch inszeniert. Die Repräsentanten einer christlichen und einer höfisch-weltlichen Ordnung treffen aufeinander und agieren die Konkurrenz aus. Doch während die Handlungsallegorie eine einfache Unterordnung der Frau Welt unter die christliche Ordnung zeigt, wird der Konflikt auf der Ebene der Ordnungsindikatoren differenzierter dargestellt. Denn das hierarchische Ordnungskonzept, das Christus der Frau Welt überordnet, ist nur eine von mehreren Ordnungsmöglichkeiten. Ausgehend von den anderen Ordnungsindikatoren steht weniger die Unterordnung als vielmehr die Transformation im Zentrum: So werden die symbolischen Ordnungsindikatoren (Kleider, Herrschaftsinsignien) durch eine christliche Symbolik umcodiert. Ähnliches lässt sich auch bei den zeitlichen und räumlichen Ordnungsindikatoren beobachten: Die zyklischen Jahreszeiten verlieren durch die Tageszeiten, aber auch durch die Frage, was bleibt, an Geltung: Während die Diener der Welt und die Personifikation selbst in den Höllenflammen zerstört werden, behält der Pilger bzw. Christus seine Macht über das Ende der Zeiten hinaus. Die irdische Zeit vergeht und an deren Stelle tritt das Endgericht. Dementsprechend wird auch der

30 WITTHÖFT, Sinnbilder, S. 193-195 (Anm. 26); für Konrad von Würzburg vgl. auch QUAST, Bruno: Lektüre und Konversion. Augustinus, Konrad von Würzburg, Petrarca. In: Geltung der Literatur. Formen ihrer Autorisierung und Legitimierung im Mittelalter. Hrsg. von Beate KELLNER u. a., Berlin 2005, S. 127-137, hier S. 133f.; KERN, Weltflucht, S. 43-68 (Anm. 9).

31 Auffällig ist, dass nur die Berliner Hs. (Abb. 2), f. 134r, Frau Welt nackt darstellt. Die Dresdner (Abb. 1), f. 75r, und die Düsseldorfer Hs. (f. 65v) zeigen sie in Kleidern, an denen Schlangen und Kröten hochkriechen. Auch die Bilder zeigen so eine Transformation der Frau Welt und nicht wie bei den Skulpturen in Worms (s. o. Anm. 28) und Straßburg (Fürst der Welt) oder bei der Fortuna anceps eine zweigeteilte Gestalt. Die Dresdner Handschrift zeigt zudem - aufgrund der für die Lauber-Werkstatt typischen Gruppendarstellungen - die Betrachter der Frau Welt (f. 71v und f. 75r.; vgl. Abb. 1) und macht so deutlich, dass es Frau Welt ohne die Weltdiener nicht gäbe. 
Raum, in dem Höfisches und Außerhöfisches differenziert werden und in dem soziale Rituale statthaben, aufgelöst: velt, anger, heide und ouch der walt / Die wurden jemerlich gestalt: / E3 wart enphlamet und enzunt (WL 241-243). An dessen Stelle werden die Schreie und Klagen der durch Christus gerichteten Weltdiener hörbar (WL 250-277). Der Raum der höfischen Freude transformiert sich so in einen Gerichts- und Strafraum.

Die Ordnungskonkurrenz wird zudem auf der Ebene der (rhetorischen) Instanzen und damit auf der Ebene der Ordnung des Erzählens ausgetragen: Die Personifikation der Frau Welt geht mehr oder minder in dem Moment in den Flammen unter, in dem sie durch die Namensnennung von einer ,gewöhnlichen' Frauenfigur zur als ,Frau Welt' bezeichneten Personifikation wird. Im Moment der vollständigen rhetorischen Entfaltung der Personifikation wird diese zerstört und ihre Macht als bloßer rhetorischer Effekt dargestellt: als begrenzt und vergänglich. Ihr Gegenspieler ist jedoch keine rhetorische Figur, sondern Christus selbst, der auch in einem Text immer mehr ist als ein bloßes Zeichen. Dadurch erhält seine abschließende Rede eine besondere Wirksamkeit: Sih miner wunden leit / Die ich durch dich enpfangen han! Waz ich uch gůtes han getan / Des hat ir mir gedancket niht (WL 288-291). ${ }^{32}$ Die Rede richtet sich vordergründig an die im Feuer schreienden Weltdiener, adressiert aber selbstredend auch - unterstützt durch die zweite Person Singular - die Rezipienten.

Die Funktion der Instanz welt ist somit im zweiten narrativen Teil des Textes eine andere als in der Ich-Rede am Beginn. Hat die adressierte welt dort verschiedene Ordnungskonzepte harmonisiert, so gilt das zwar im zweiten Teil weiterhin. Darüber hinaus wird ihr rhetorischer Status als Personifikation aber auch dafür genutzt, das mit ihr verknüpfte Ordnungs- und Symbolsystem zu depotenzieren.

Die Erzählung endet mit den Schreien der verdammten Weltdiener und der Antwort von Christus. Die Weltdiener bitten um erbarmherczikeit (WL 251, 261), erhalten aber gereht gerihte (WL 269) bzw. gerehtikeit (WL 281). ${ }^{33}$ Christus schließt die Möglichkeit der bůz explizit aus (WL 298) und verheißt den Rezipienten,

32 In Handschrift K wird nicht der Einzelne, sondern die Gruppe angesprochen: Die ich durch uch enpfangen han (Otto, der slecht weg, S. 509, V. 341 [Anm. 11]). Damit wird auf die für die Konzeption des Endgerichts zentrale Passage im NT angespielt, Mt. 25,31-46. Auch das Zeigen der Wunde ist typisch für textuelle Darstellungen vom richtenden Christus; vgl. dazu ScHWARZ, Reinhard: Die spätmittelalterliche Vorstellung vom richtenden Christus - ein Ausdruck religiöser Mentalität. In: Geschichte in Wissenschaft und Unterricht 32,1 (1981), S. 526-553, hier S. 537 f. 33 Dem entspricht auch die im Vergleich mit Konrad von Würzburg, Der Welt Lohn (Anm. 25), andere Deutung des sogenannten lon[s] der welt. Dieser ist nicht die eigene Vergänglichkeit bzw. die Vergänglichkeit des irdischen Reichtums (V. 214-238), sondern der Entzug der Freuden des Himmelreichs (WL 235) bzw. die Verdammnis (WL 296f.). 
die uf der schanden pfat gehen, denselben lon wie den bereits verbrannten Weltdienern (WL 293-296). Damit wird abschließend sichtbar, worauf die christliche Ordnung gründet: Auf der Sanktionsmacht des göttlichen und damit des außerweltlichen Gerichts. Indem der Wunsch nach erbarmherczikeit aber dennoch so viel textuellen Raum erhält, wird zugleich eine Ordnungskonkurrenz innerhalb der christlichen Ordnung sichtbar: Gründet die christliche Ordnung auf Barmherzigkeit oder auf der gerechten Strafe ${ }^{34}$ Die Rede von Christus stellt hier allein die gerechte Strafe in den Vordergrund. In der daran anschließenden Mahnrede wird diese Frage wieder aufgegriffen, dann aber differenzierter beantwortet.

Im zweiten, erzählenden Teil des Textes wird Ordnung somit als eine prozessuale entworfen. Die erzählte höfisch-irdische Ordnung wird (auf der Ebene der Darstellung) nicht wie in der Ich-Rede gesetzt, sondern anhand von Ordnungsindikatoren entwickelt und transformiert: Die höfischen Ordnungsindikatoren sowie die damit dargestellte Ordnung werden durch eine höhere, christliche Ordnung relativiert und überformt. Vorgeführt wird so nicht nur die höherrangige Geltung der christlichen Ordnung, sondern auch deren Bezugnahme auf die sog. weltliche Ordnung, die sie relativiert und transformiert. Die vom Text etablierte christliche Ordnung ist deshalb nicht nur auf der Ebene des Erzählens eine prozessuale, weil sie im Verlauf des Erzählens entsteht; sondern sie ist auch auf der Ebene des Erzählten eine prozessuale, weil ihre Geltung sich der Transformation und Überwindung der zuerst etablierten höfisch-irdischen Ordnung verdankt.

\section{Transformation und Überblendung von Ordnungen (Mahnrede)}

Auf die ca. 200 Verse lange Ich-Erzählung folgt eine Mahnrede von ca. 350 Versen. Wann genau die Erzählung bzw. die Rede von Christus aufhört und wann die Mahnrede einsetzt, ist nicht eindeutig feststellbar. Entweder geht die Rede von Christus sukzessive in die eines Predigers über ${ }^{35}$ oder man setzt den Beginn

34 Theologisch sind selbstredend Gerechtigkeit und Gnade nicht voneinander zu trennen, allerdings wird die Verknüpfung unterschiedlich konzipiert; vgl. dazu SCHARBERT, Josef u. a.: Art. ,Gerechtigkeit‘. In: Theologische Realenzyklopädie 12, Berlin 1984, S. 440-448, hier S. 416, 419. Zudem werden in der Liturgie der barmherzige und der richtende Christus z. B. im Rahmen der Adventssonntage eng aufeinander bezogen; vgl. ScHwaRz, Vorstellung, S. 538f. (Anm. 32).

35 In Hs. K gibt es bei WL 299 ein Paragraphenzeichen (Отто, der slecht weg, S. 509, V. 329 [Anm. 11]). Versteht man dies als Markierung einer neuen Redeeinheit, dann spricht hier und im Folgenden noch Christus. Allerdings wird in WL 316 got angerufen und um ewige Freuden 
der Mahnrede bei WL 301, wenn im predigtartigen wir auf die Erzählung wie auf ein Gleichnis oder Bispel Bezug genommen wird: Wir han der welte hof gesehen / und allez daz sie hat verjehen / und offenliche hat geseit (WL 301-303). Danach wechseln sich Erklärungen zur erzählten Geschichte, Gegenwartsklage, gebetsartige Bitten, Mahnungen und Drohungen ab. So wird die Gerichtssituation nicht nur erneut thematisiert, sondern ebenso deutlich gemacht, dass diese auch auf die Rezipienten zukommen wird: Nu han wir alle wol vernomen, / Daz wir zů hofe müzen komen, / Den nu der here halten wil (WL 343-345). Doch statt wie im narrativen Teil ganz auf die Androhung der Strafe zu setzen, wird hier die Belohnung thematisiert. ${ }^{36}$ Die Auserwählten und die Möglichkeit ewiger Freuden werden beschrieben (WL 371-388) und es wird gefragt, wie der Einzelne dies erreichen kann. Der Weg zu Gott sei steinig und die Zeit kurz (WL 389-412). Wichtig sei es deshalb, sich der künftigen Gerichtssituation (dem rechenunge geben; WL 409) immer bewusst zu sein. Von der Sanktionsmacht, aber auch Belohnungsinstanz ,Gericht‘ kommt der Text so auf die Haltung bzw. die richtigen Erwartungen der einzelnen Gläubigen zu sprechen. Die göttlich-jenseitige Ordnung wird so über die Erwartung des Einzelnen, der um das Endgericht weiß, auf die innerweltliche Ordnung bezogen. Dies prägt auch den Argumentationsgang der Rede. So wird angekündigt, dass der here die vier pfifer (apokalyptischen Bläser bzw. Engel ${ }^{37}$ ) auffordert, zwischen den Auserwählten und den Verdammten zu unterscheiden bzw. zwischen denen, die zů rehte sint becleit (WL 372), oder denen, die cleider hant / Die niht dem hofe wol an stant (WL 375f.). Von diesen himmlischen Kleidern ausgehend kommt das Prediger-Ich auf innerweltliche Kleidersitten zu sprechen (WL 413-452). Beklagt wird, dass mit freizügiger Kleidung (von Frauen und Männern) um Aufmerksamkeit beim jeweils anderen Geschlecht geworben wird. Dabei thematisiert das Prediger-Ich auch, ob seine Ermahnungen bei den Rezipienten Gehör finden. Es beklagt, dass allzu häufig den Falschen zugehört und falsche Ratschläge befolgt würden (WL 453-460). Dadurch werde das Leben auf der Basis falscher Erwartungen (bo̊se zůversiht; WL 461-466) geführt. Der Text sieht seine eigene Funktion somit nicht zuletzt darin, die Erwartungen der Rezipienten richtig auszurichten. ${ }^{38}$

gebeten. Der Übergang von der Rede von Christus in die Rede eines Prediger-Ich wäre dann fließend.

36 SchwARz, Vorstellung, S. 539 (Anm. 32), geht davon aus, dass im Spätmittelalter vermehrt nicht mehr nur die Bestrafung, sondern auch die Belohnung hervorgehoben wird.

37 Auch die vier Engel in der Rolle von Gerichtsdienern gehören zur typischen Endgerichtsdarstellung dazu; vgl. SCHWARZ, Vorstellung, S. 537 (Anm. 32); BRANDENBURGER, Egon u. a.: Art. ,Gericht Gottes‘. In: Theologische Realenzyklopädie 12, Berlin 1984, S. 459-497, hier S. 488.

38 Vgl. dazu auch WL 313-315, 651-655. 
Während der narrative Mittelteil die Ordnungskonkurrenz zwischen einer christlichen und einer weltlichen Ordnung allegorisch darstellt, diskutiert die Mahnrede, was der Wechsel von einer weltlichen zu einer christlichen Ordnungserwartung innerweltlich bedeuten könnte. Dabei verfährt die Mahnrede im Bezug zur Erzählung weder rein allegorisch-deutend, noch leitet sie aus dem narrativen Geschehen konkrete Verhaltensregeln ab. Vielmehr geht es dem Text darum, die innerweltlichen Erwartungen des Rezipienten umzugestalten: Die Rezipienten sollen die diesseitige christliche Ordnung unter der Erwartung der jenseitigen christlichen Ordnung betrachten, dann stellt sich auch das richtige Handeln ein.

Die Ordnungstransformation, die wir im narrativen Mittelteil beobachtet haben, wird in der Mahnrede ebenfalls fortgesetzt - jedoch nicht auf der Ebene der Ordnungsindikatoren, sondern auf semantischer Ebene. Die Mahnrede beginnt damit, dass sie - eine Rede des Pilgers aufgreifend ${ }^{39}$ - Turnier und Fest als der welte hof (WL 301) bezeichnet. Vierzig Verse später bezieht sich der Sprecher erneut mit dem Wort hof auf die Erzählung, doch geht es nun um den hof des Herrn: Nu han wir alle wol vernomen / Daz wir zů hofe müzen komen. / Den nu der here halten wil (WL 343-345). Der welte hof ist nicht zerstört oder unterjocht worden, sondern hat sich in die Gerichtssituation, den göttlichen Gerichtshof am Ende der Zeit verwandelt. Bevor jedoch die Rezipienten zů dem hofe ge[n] (WL 361), sollen sie Gott bitten, dass wir doch wol zů hofe komen / E wir die pfifen han vernomen (WL 501f.). Danach werde Des hofes reht, des heren zorn (WL 518) herrschen. Die Rezipienten erscheinen so als ,auf dem Weg zum hof des Herrn'. Deshalb müssen ihre innerweltlichen Handlungen und Erwartungen auf die Gerichtssituation ausgerichtet sein. Anhand der Semantik von hof werden somit die innerweltliche Ordnung und die jenseitige christliche Ordnung überblendet, wobei das Endgericht den letztgültigen Fokuspunkt darstellt. Die sozial-irdischen Konnotationen des Wortes hof werden dabei auffällig präsent gehalten: Neben des hofes reht (WL 518) geht es auch darum, des hofes siten einzuhalten (WL 644), und zum hofe muss man, bereit und zư rehte becleit sein (WL 371f.). Die Überblendung von irdischem und göttlichem hof hat somit in beide Richtungen statt. Nicht nur soll hinter der irdischen Ordnung das göttliche Gericht aufscheinen, sondern dieses wird auch mit Verweis auf innerweltliche soziale Institutionen und Praktiken konzipiert. ${ }^{40}$

39 Der Pilger spricht bereits früher davon Daz man dem hofe ein ende git (WL 176); davor spricht auch der Erzähler vom hof ] der frowe (WL 166).

40 Nicht selten finden sich an sog. weltlichen Gerichtsorten (Ratshäusern usf.) Endgerichtsdarstellungen und umgekehrt sind die Endgerichtsdarstellungen von irdischen Gerichtssitten geprägt; vgl. ScHWARZ, Vorstellung, S. 532-534, 544 (Anm. 32). Hier geschieht dies allerdings nicht auf der Basis der Semantik von Gericht, sondern von hof. 
Auch die am Ende des narrativen Mittelteils aufscheinende Frage nach der Barmherzigkeit Gottes bzw. der gerechten Strafe (WL 259-298) wird dabei nochmals aufgegriffen: ${ }^{41}$ Der einzelne Rezipient wird $\mathrm{zu}$ Beichte und Buße ermuntert und es wird ihm - wegen Gottes erbarmherczikeit (WL 489, 503) versprochen, dass er von seinen Sünden befreit werden kann (WL 486-502). Kommt aber der Einzelne niht gelutert gar (WL 508) - also ohne Sündenfreiheit zum Gericht (WL 507-528), dann erfährt er des heren zorn (WL 518). Hie [im sündenpfưl] enist keine erbarmherczikeit (WL 516). Die Frage nach dem Verhältnis von Gottes Barmherzigkeit und gerechter Strafe wird somit wie in vielen katechetischen Texten der Zeit ${ }^{42}$ dadurch gelöst, dass Gottes Barmherzigkeit die Möglichkeit der Reue und Buße eröffnet; wenn diese jedoch nicht genutzt wird, die ewige Verdammnis droht. Für den einzelnen Gläubigen hat dies zur Folge, dass er die Zeit, die er hat, nicht mit unstetigkeit (WL 527) vertrödeln darf, sondern sie für Reue, Beichte und Buße nutzen muss (WL 521-528). Zugleich entsteht der Eindruck, dass die christliche Ordnung, um die es der Erzählung geht, sich gerade durch die Erwartung von Barmherzigkeit und gerechter Strafe auszeichnet. ${ }^{43}$ Die Bezeichnung des Endgerichts als hof, könnte genau dies konnotieren: Beschrieben wird eine sowohl sanktionierende als auch barmherzige, sowohl strafende als auch fürsorgliche Institution. ${ }^{44}$

Im Anschluss an den Aufruf zu Reue beginnt im Text unvermittelt ein reuiges Sünder-Ich zu sprechen (WL 529-558). ${ }^{45}$ Seine Rede bietet den Rezipienten ein Muster, mit dem sie zu Reue und Sündenbewusstsein, also zur richtigen Haltung und $\mathrm{zu}$ richtigen Erwartungen, finden. Mittels der Klage eines betroffenen, ebenfalls sündigen Ich wird daran anschließend die mangelnde Ehrfurcht gegenüber alten Menschen gescholten (WL 559-595). Die am Beginn für den gesamten Text

41 Die zwölf Ritter der Erzählung werden als zwölf Apostel gedeutet, die die Sünder bekehrt und zu Gott geführt haben (WL 321-330). Dadurch habe Gott gerehtikeit vollbracht (WL $329 \mathrm{f}$.). 42 SCHWARZ, Vorstellung, S. 539-541 (Anm. 32).

43 Dementsprechend wird in katechetischen Texten betont, dass die Barmherzigkeit des Richters darin besteht, dass die Höllenstrafen milder und die Belohnung größer ist als verdient; vgl. SCHWARZ, Vorstellung, S. 546 f. (Anm. 32).

44 FouCaulTs Konzept der Pastoralmacht ist sicherlich historisch und kirchengeschichtlich genauer zu differenzieren. Wenn man sich jedoch fragt, weshalb hier die Semantik von hof so dominant ist, könnte es gleichwohl die Antwortrichtung angeben: FoucAULT, Michel: Sécurité, territoire, population. Cours au Collège de France (1977-1978). Hrsg. von M. SENELLART, Paris 2004, S. 167-260.

45 Dieses Ich bereut seine Sünden (WL 529-558), klagt die welt an und beklagt, dass es sich wegen des Strebens nach weltlichen Gütern versumet (WL 547, 554) hat - also die Zeit vergeudet hat. 
festgestellte Mehrstimmigkeit zeigt sich somit in der Mahnrede nochmals intensiviert. ${ }^{46}$ Sie ermöglicht, verschiedene Perspektiven einzubringen und das Thema zugleich aus abstrakter wie konkreter Perspektive zu diskutieren. Die Rezipienten erhalten so unterschiedliche Identifikationsmöglichkeiten und werden in unterschiedlicher Weise adressiert.

Auch in diesem dritten Teil des Textes werden somit Ordnungen dargestellt und verhandelt. Doch geht es nicht mehr um die Frage, welcher Ordnung und welchen Ordnungsindikatoren welche Geltung zukommt, sondern wie jenseitige und diesseitige christliche Ordnung anhand der Erwartungen des Einzelnen ineinander verfugt sind. Anders als die in zwei Handschriften (B und D) anschließende Dekalogparaphrase ${ }^{47}$ und die in vier Handschriften vorausgehende Versauslegung der Zehn Gebote ${ }^{48}$ vermittelt die Mahnrede daher kaum Normen. Sie zielt auf eine innerweltliche christliche Ordnung, die nicht bzw. nicht nur auf Regeln und jenseitigen Sanktionen gründet, sondern die durch die richtigen (Endgerichts-)Erwartungen bereits innerweltlich erzeugt wird. Die Mahnrede bezeichnet dies als cristenliche[ ] zůversiht (WL 639), die sie den falschen Erwartungen (bo̊se[n] oder krancke[n] zůversiht; WL 463), ${ }^{49}$ die weltliche Werte wecken, entgegenstellt. Diese cristenliche[ ] zůversiht (WL 639), ist zugleich aber auch das hoffnungsvolle Pendant zur grimme[n] zůversiht (WL 529, vgl. JC 51): zur Erwartung des unbarmherzig strafenden Gottes.

Damit zielt die Mahnrede auf das ab, was wir im narrativen Mittelteil bereits anhand der Ordnungsindikatoren beobachtet haben: Weltlich-höfische Erwartungen sollen in christlich eschatologische transformiert werden. Neu wird dafür in der Mahnrede jedoch das Mittel der Überblendung eingesetzt: Die weltliche und die christliche, die diesseitige und die jenseitige Ordnung sollen wie der hof der welt und der hof des heren als ineinander verfugt erkannt werden.

$46 \mathrm{Zu}$ dieser Mehrstimmigkeit trägt auch die relativ lange Passage bei, in der die Rede des here zu den pfifer[n] widergegeben wird (WL 370-388). Auch in dieser Passage ist zwar der Einsatz, nicht aber das Ende der Rede klar bestimmbar.

47 Siehe oben Anm. 21.

48 Der Text, der dem Weltlohn vorausgeht, ist eine Kompilation aus den Zehn Geboten, den Sieben Sakramenten, den Sechs Werken der Barmherzigkeit, den Sieben Tagzeiten, Verse vom endechrist und den Sechs liturgischen Farben. Es handelt sich also um eine Sammlung von Normen, Geboten und heilsgeschichtlichem Wissen. Die Aufzählung deutet darauf hin, dass es hier um grundlegende Normen und Wissen geht, das sich alle einprägen sollen. Allenfalls wegen einer Lagenverwechslung weicht die Reihenfolge in Hs. B von der der anderen Hs. ab. Vgl. Oтто, der slecht weg, S. 468-498 sowie 570-572 (Anm. 11).

49 Vgl. auch JC 59. 


\section{Fazit}

Im Unterschied zu den benachbarten normativen Kotexten, die wie der Dekalog Regeln vermitteln, konzipiert unser Text die innerweltliche christliche Ordnung als eine, die durch Erwartungen bzw. durch rechte cristenliche[ ] zůversiht (WL 639) performativ erzeugt wird. Die Frage nach den rechten Erwartungen wird im ersten Teil des Textes aus der Perspektive desjenigen, der durch weltliche Werte enttäuscht worden ist, eingeführt. Im narrativen Mittelteil wird allegorisch, anhand der Konfrontation der Frau Welt mit Christus, eine Wertekonkurrenz zwischen sog. weltlichen und christlichen Werten dargestellt. Diese Ordnungskonkurrenz wird jedoch nicht so sehr als Rangwettstreit ausgetragen, sondern sie wird in einen Transformationsprozess überführt: Die höfischen Ordnungsindikatoren werden christlich umcodiert. Dementsprechend sollen die Rezipienten lernen, ihre weltlichen Erwartungen durch christliche Erwartungen und damit durch die Erwartung des Endgerichts zu überwölben. Auch in der Mahnrede ist dies wiederum Thema, wenn den Rezipienten nahegelegt wird, hinter dem hof der welt den hof des here zu erkennen. Innerweltliche und jenseitige Ordnung werden anhand der Semantik von hof nicht nur aufeinander bezogen, sondern geradezu überblendet.

Der dreiteilige Text versucht somit zu einer christlichen Ordnung hinzuführen, indem er die Rezipienten an die Erwartung des Endgerichts gewöhnt. Dazu werden auch die Ordnungen des Erzählens genutzt: Die formale Heterogenität der drei Textteile, die Vielstimmigkeit der Ich-Rede sowie die prozessuale Darstellungsform im Mittelteil bieten vielfältige Identifikationsmöglichkeiten und schulen über die intratextuellen Erwartungen auch die extratextuellen. Das Besondere an der dadurch entworfenen erzählten bzw. dargestellten Ordnung ist nicht, dass sie nur ansatzweise über Ordnungsindikatoren, Figuren-Erwartungen oder präskriptive Aussagen greifbar wird - das gilt für fast alle erzählten Ordnungen. Das Besondere ist vielmehr die Zweistufigkeit der dargestellten Ordnung und damit auch der Erwartungslenkung. Das Endgericht gehört einer jenseitigen Ordnung an, die die Rezipienten im Diesseits erwarten müssen, damit eine diesseitig-christliche Ordnung entstehen kann. Diese (in sich gedoppelte) christliche Ordnung ist zudem, so zeigt der narrative Mittelteil, nur als Transformation einer zu überwindenden weltlichen Ordnung darstellbar. Der Weltlohn thematisiert so eine performativ konzipierte christliche Ordnung mit den Mitteln eines Textes, der in seinem Verlauf Ordnung(en) unterschiedlich entwirft und transformiert. Die Prozessualität der dargestellten Ordnung konvergiert so mit der Prozessualität der Ordnung des Darstellens. 